\title{
Principles of Diagnosis in Neurology
}

\author{
J. CLIFFORD RICHARDSON
}

It is important and interesting to reconsider principles of diagnosis in neurology in the light of the much expanded knowledge of pathogenesis and treatment, and the vast array of new diagnostic and investigative machinery. This is a rather dazzling scene that includes advanced new techniques of radiology, applied electronic neurophysiology, and sophisticated biochemical estimations. Enzymes and neurotransmitters come into the picture, and the computer is evident everywhere. There is a host of diagnostic procedures, old and new optical, otological, psychological, genetic and pharmacological. And very much in evidence are those persons behind the machines radiologists, physiologists, pharmacologists, otologists, ophthalmologists and pathologists. They each deserve and enhance the prefix "neuro". In this complicated spectrum of modern clinical neurology one can still see the neurologist and neurosurgeon remaining prominently in view, though their halos have dimmed.

In venturing towards my subject with a little trepidation, I was reminded of an experience of Harvey Cushing when he spoke to a luncheon of The Association for Research in Nervous and Mental Disease in 1938. It was a large audience with a preponderance of researchers. He said:

Dr. Richardson was a distinguished guest of the 11 th Annual Congress of Neurological Sciences held at Winnipeg, October 13-16, 1976. This paper is his address to the Congress.

Reprint requests to Dr. J. Clifford Richardson, 305170 St. George St., Toronto, Ontario, Canada M5R 2M8.

\begin{abstract}
My theme is not exactly "Back to Hippocrates" for that is a long way to go so soon after lunch, and yet in a sense that is just what it is. For could the Father of Medicine have foreseen that one of its branches was some day to concern itself solely with the nervous system and its disorders, he would have added this further aphorism: "Should you incline to pursue that obscure but important subject called neurology, and wish to succeed therein, CULTIVATE YOUR POWERS OF BEDSIDE OBSERVATION."
\end{abstract}

He felt that his speech had been a failure and lamented to a friend the next day that his remarks had been followed by profound silence. Fulton, his biographer, kindly adds that the silence had not indicated disapproval but that his audience was reflecting on the challenge of this message.

In all events I shall attempt a review and reconsideration of the basic clinical techniques of neurological diagnosis in the light of the many available ancillary procedures. I chose this ambitious topic when in London at the Holmes Centennial Meeting earlier this year. My point of view is greatly influenced by Sir Gordon because of an apprenticeship with him forty years ago. And his memory has been livened very much by this recent symposium. In fact, all owe a great deal to Holmes for clinical studies and teaching which provided the methods of examination still generally in use today. The outstanding and lasting contribution of Holmes was his series of classical investigations of physical signs of neurological disease in man. Notable were his studies of sensation with Head, and of the cerebellum and visual system based on brain wounds studied dur- ing World War I. He discovered and taught that the elicitation of scientific data at the bedside required a discipline of method as strict and rigid as in the laboratory. Moreover, he found that he could maintain his own expert clinical skill only through constant and repeated use. Holmes wrote of Hughlings Jackson: "Much of his teaching is embodied in the corpus of modern neurology.' Now a half century later that statement describes equally his own (Gordon Holmes) teaching.

The clinical diagnosis may be considered founded on three basic pillars - history taking, neurological examination, and clinical deduction, or deductive analysis. The deductive process starts with an interpretation of the elicited symptoms, including signs. They can be indicative of a direct, positive or negative effect of the lesion, of indirect release effects or reorganization of uninjured mechanisms. One proceeds then to further logical reasoning about the anatomical site of the lesion and then to final conclusions as to the type and nature of such pathology and morbid physiology. Cautious adherence to these basic principles often permits diagnosis without going beyond the personal confrontation with the patient, combined with analytical reasoning. In fact a large proportion of office neurological consultations can provide a sound opinion without further aids. Too often, however, the history and examination are rushed and serve as mere stepping stones to a seductive list of procedures - X-rays, E.E.G., E.M.G., isotope scan, C.T.T. scan, and what not. Because these procedures are both interesting and available, it requires thought and discipline to avoid using them extravag- 
antly. The viewpoint of keeping ancillary procedures to a minimum is supported by preferences for logical scientific reasoning as well as for economy. This obvious problem has been pointed out quite often. About fifteen years ago in Saskatoon the late Professor Allan Bailey presented to a provincial general medical meeting a thoughtful and nicely phrased lecture in which he was sharply critical of a tendency to excessive and precipitous use of x-rays, E.E.G.'s and other procedures in dealing with common neurological problems. My own remarks here are justified by the ever increasing danger of a hurried mechanical sort of "go-go neurology'.

There is no need to pursue a campaign of protecting the neurologist as a threatened species, but certainly it is essential that we critically review our basic techniques to see that they remain valid and up-to-date in the contemporary neuroscience world. Let us then have a look at those three fundamentals - history taking, examination and deductive analysis.

\section{History Taking}

History taking in neurology must remain basic for diagnosis and for the acquisition of new knowledge. The greatness of master neurologists like Sir Charles Symonds was in large measure due to cultivated skill and meticulous care in obtaining and recording the history. Even with all our new aids, it remains true that the solution to some tricky diagnostic problems often is found by careful detailed retaking of the history. Abbreviation of this essential diagnostic discipline in favor of time saving procedures may serve to answer quickly some urgent therapeutic problems, but it does not help to provide a full understanding of the clinical situation. It should be remembered that the human patient is a superior research model because he has the faculty of speech and much is lost in clinical investigation if subjective historical data are not fully revealed.

Good history taking includes an evaluation of the emotional aspects of illness and a sorting out of the "organic" and the "functional". The assessment of pain requires a gauging of the patient's reaction, be it realistic, minimizing or exaggerating. Proficiency in these and many other diagnostic niceties are the requirements of a competent neurologist. Assessment of the severity, validity and implication of a patient's pain is a vital and basic principle of diagnosis. Very often the sound evaluation of a neurological symptom, particularly pain, is the chief determinant for or against a surgical operation. Help is sought from psychological tests, psychiatric interviews, electrophysiological procedures, and examination during barbiturate narcosis. But, in the end the decision rests on the judgment of the attending clinician, and certainly he is not always a neurologist. The wise and experienced doctor may be neurosurgeon, orthopaedic surgeon, internist or family physician. It is difficult to suggest worthwhile major revision of the traditional old methods of history taking. Innovations such as the Problem Orientated Record System have suggested modifications of debatable value. To be sure, discoveries about genetic factors, drug induced symptoms and industrial poisons, add subjects for clinical inquiry.

In the study of medicine, one learns history taking by rather informal precept and practice, and this is probably best. In interviewing, or examination by interrogation, we have much in common with other professions, particularly legal, and behavioral scientists. Medico-legal experience has impressed me with the skill and formality of the barristers and judges in their methods of obtaining, in their pretrial examinations and in court procedures, reliable information by their interrogation of witnesses. I have wondered if we might steal a leaf from their book by making greater use of legal doctrine such as formal rules of evidence. However, it is wise to avoid any adoption of the harsh adversary system of justice, the better to continue our own techniques of searching out the truth. It seems appropriate to continue with a medical his- tory taking, largely by rule of thumb, while remembering the degree to which this method may be perfected by care and experience.

\section{Examination}

With regard to neurological examination one feels less complacent with the old methods which have changed so little in the past fifty years. With all the new knowledge about special senses, motor functions and sensation, are we adequately up-dating these clinical methods?

The general plan and basic techniques of neurological examination withstand the test of time and have changed little. It seems that the examination of higher to lower functions - mentality and speech, cranial nerves, motor functions, reflexes and sensation - is a pattern to be followed in perpetuity. Looking for modern trends in routine neurological examination, one can see both positive and negative effects of rapidly advancing neurological scientific knowledge. Mental examination is carried out as well or better than in the past. There have been some useful simplifications of aphasia testing. Visual field examination and charting have become improved with new perimeters and techniques, though this has resulted in a tendency for the general neurologist to neglect or lose skill with the tangent screen and simple perimetry. Other techniques of visual and oculomotor testing have been expanded, with more frequent use and understanding of opticokinetic nystagmus, cold water calorics, gaze palsies and special pupillary reactions. Only minor frills have been added to motor and reflex examination, but probably we can look for useful new contributions from many busy and productive applied neurophysiologists. Sensory examination has taken on certain parietal lobe tests as well as some new quantitative techniques, but nothing will replace the simple older techniques as perfected by extensive painstaking practice, supported by careful sensory charting. We have become more meticulous and expert in examination for neck and head 
bruits. A special testing of brainstem reflexes in states of coma has been adopted, widely and usefully. Altogether, there is an obvious need both for fence mending to maintain proven older skills, and for fresh initiative in applying new knowledge.

Though the routine general neurological examination has changed little, there have been great advances in diagnostic facilities and techniques by the special specialists neuro-ophthalmologists, otologists, neuropsychologists, the neuromuscular neurologists, and others. For examination in depth as required in complex diagnoses, there has evolved a highly effective team approach. We have learned not to scoff at "diagnosis by committee" and to welcome, to encourage, such collaborative effects. Neurological problems can be so complicated that assessment can be accomplished only by parcelling out the work to consultants and therapists.

However, the attending neurologist or neurosurgeon should have sufficient knowledge and training in special diagnostic procedures to observe and discuss the test results, not merely glance casually at the final opinion in the report of his special colleague. Good practice requires of the responsible clinician that he look at the E.E.G. tracings, the pathological slides, and the protocols of the psychologist and electromyographer. Here is the challenge of a continuing neurological education, in realizing that it is impossible to become expert in all of these fields.

\section{Deductive Analysis}

The third step in clinical diagnosis, the deductive analysis after history and examination, presents exciting and challenging new perspectives. It remains sound and essential to interpret the elicited symptoms in terms of anatomy and physiology, then to think of pathology. Such clinical reasoning is crucial to rational therapy. Here, particularly, we see the intellectual fascination of neurology. Nowadays, there is a tremendous amount of new factual knowledge to be marshalled together as one correlates clinical findings with disturbed physiology. Cerebellar ataxia, extrapyramidal rigidity and tremor, for example, have new meanings in terms of cerebellar circuitry, the organization of higher centres of motor control, and muscle spindle physiology.

In the interpretation of abnormal movements like chorea, dystonia, myoclonus and facial dyskinesia, we are presented with a new conception by the pharmacological discoveries of related neurotransmitter dysfunction. Such new views derive from studies of drug induced dyskinesia, Parkinson's disease since the dopamine era, hypoxic myoclonus and serotonin precursor treatment, and perhaps GABA studies in Huntington's disease. Now, in observing abnormal movements it is appropriate to attempt deductions in such concepts as dopamine depletion, dopamine receptor hypersensitivity, and damage to serotonergic neurones. The approach to dystonias, including spasmodic torticollis, may become similarly modified.

The scope of deductive analysis in terms of pathology is vastly widened by our new knowledge. Older convenient classifications of disease, such as the system degenerations (cortical, extrapyramidal, pyramidal and cerebellar), and multisystem degenerations (such as Parkinsondementia), are becoming passé with discoveries related to slow viruses, enzyme faults, unsuspected drug causation and transmitter disorders. Perhaps one may look forward to an eventual disappearance of the word "degeneration" in neuropathology. I shall not attempt description of many new diseases and syndromes being brought to light by the enzyme chemist, geneticist and child neurologist.

This discussion of basic clinical techniques is presented with full appreciation of the great and growing superstructure of laboratory and other ancillary procedures. The stock in trade of the neurologist is vastly increased and enormously complicated. A judicious under- standing and reasoned usage of these special diagnostic techniques is a major responsibility of the clinician. In fact, there is need for a secondary set of principles for guidance in these neurodiagnostic procedures. A first principle must be therapy. For example, cerebral angiography is seldom justified unless it promises information essential to further treatment. Secondly, special diagnostic procedures are sometimes justified to gain important new knowledge, if the risk is not too great. Thirdly, it may be valid to pursue diagnostic procedures purely to allow assessment for reasons of litigation or pension. To be avoided is the pursuit of extensive special studies merely to allay the fears of anxious patients and their kin.

In this talk on principles rather than practice of diagnosis, I shall not review the indications for, and merits of, the various procedures. Without doubt the CT scan has proved revolutionary by reason of its informative value and its noninvasive nature. It has reduced the number of hospital admissions, and it has largely precluded some other procedures such as pneumoencephalography.

Only time will tell the eventual influence on the practice of medical and surgical neurology of the many new innovations of the $x$-ray department, the laboratory and the computer. Perhaps, some of the early techniques of history taking and examination will become obsolete. However, I venture to predict that these new machines and advances will in large measure provide new incentives and leads towards retention and elaboration of the inquiry and examination carried out by direct confrontation of physician and patient.

In my essay it is not hard to see a bias towards a preservation of clinical techniques. No apology is necessary because there is an obvious need for thought and effort to maintain something of the Art of Medicine. It might be concluded that along with neuroscientists, we need neuroartists, or perhaps too, artistic neuroscientists. 